\title{
Factors Governing the Bridging Water Protonation State in Polynuclear $\mathrm{Mg}^{2+}$ Proteins
}

Cédric Grauffel ${ }^{*}, \dagger$ and Carmay Lim ${ }^{*}, \dot{\dagger},+$

'Institute of Biomedical Sciences, Academia Sinica, Taipei 11529, Taiwan

${ }^{*}$ Department of Chemistry, National Tsing Hua University, Hsinchu 300, Taiwan

*E-mail: cedric@ibms.sinica.edu.tw or carmay@gate.sinica.edu.tw; Telephone: 886-2-2789-9043

Supplementary Figure S1: Reproduction of 'PUNREV' X-ray geometry with the B3-LYP/631+G(3d,p) method. The experimental values taken from the CSD entry are given into brackets. Carbon, oxygen, and nitrogen atoms are displayed in green, red and blue, respectively. Figure generated using PyMOL.

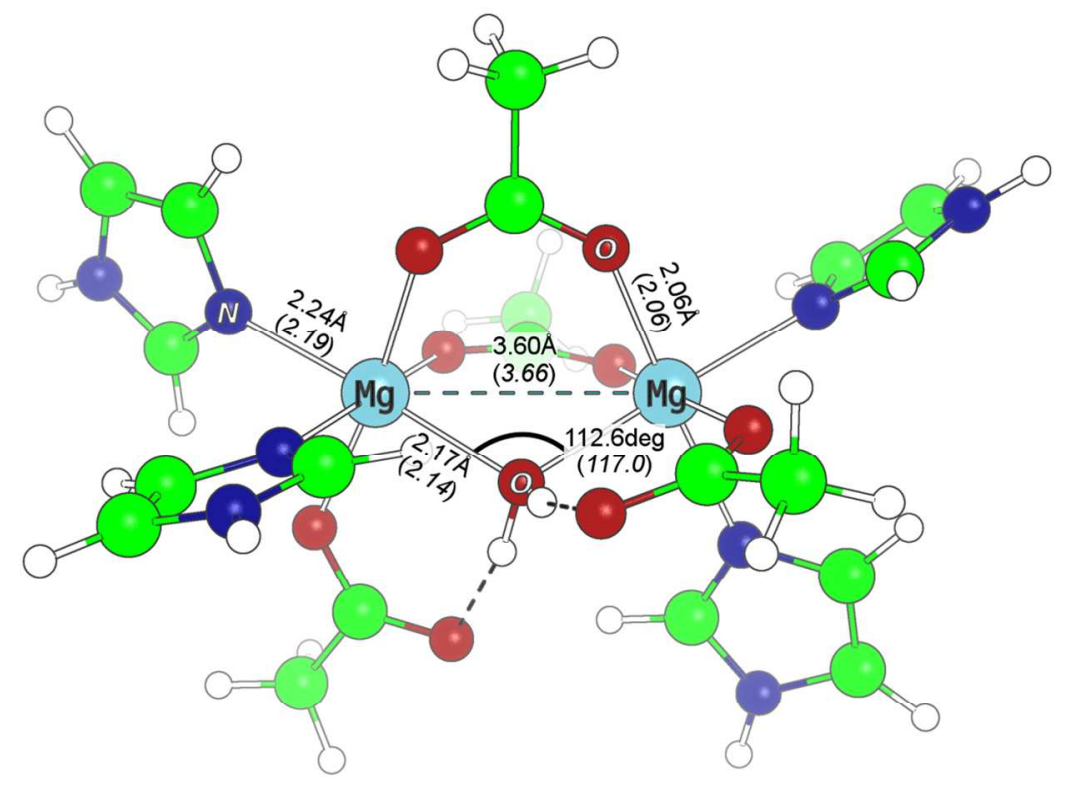


Supplementary Table S1. Comparison of mean distances to $\mathrm{Mg}^{2+}$ as well as mean angles at the bridging water $\mathrm{O}$ and $\mathrm{Mg}^{2+}$ in the 'PUNREV' $\mathrm{X}$-ray structure (numbers in italic) with those computed using B3LYP and M062X functionals and different basis sets. ${ }^{\text {a }}$

\begin{tabular}{|l|r|r|r|r|r|r|}
\hline Method & $\mathrm{Mg}-\mathrm{Mg}$ & $\mathrm{Mg}-\mathrm{N}$ & $\mathrm{Mg}-\mathrm{O}_{\mathrm{COO}}$ & $\mathrm{Mg}-\mathrm{O}_{\text {wat }}$ & $\mathrm{Mg}^{-\mathrm{O}_{w a t}-\mathrm{Mg}}$ & $\mathrm{X}-\mathrm{Mg}-\mathrm{X}^{\mathrm{b}}$ \\
\hline X-Ray: & 3.66 & 2.19 & 2.06 & 2.14 & 117.0 & 89.5 \\
\hline B3LYP/ & $\mathbf{3 . 6 0}$ & $\mathbf{2 . 2 4}$ & $\mathbf{2 . 0 6}$ & $\mathbf{2 . 1 7}$ & $\mathbf{1 1 2 . 6}$ & $\mathbf{9 0 . 0}$ \\
\cline { 2 - 7 } 6-31+G(3d,p) & $(-0.06)$ & $(0.05)$ & $(0.00)$ & $(0.03)$ & $(-4.4)$ & $(0.5)$ \\
\hline B3LYP/ & 3.62 & 2.23 & 2.07 & 2.17 & 112.9 & 90.0 \\
6-31+G(2d,2p) & $(-0.04)$ & $(0.04)$ & $(0.01)$ & $(0.03)$ & $(-4.1)$ & $(0.5)$ \\
\hline B3LYP/ & 3.64 & 2.25 & 2.08 & 2.18 & 112.9 & 90.0 \\
6-311++G(d,p) & $(-0.02)$ & $(0.06)$ & $(0.02)$ & $(0.04)$ & $(-4.1)$ & $(0.5)$ \\
\hline B3LYP/ & 3.62 & 2.25 & 2.08 & 2.17 & 112.7 & 90.0 \\
6-31+G(d,p) & $(-0.04)$ & $(0.06)$ & $(0.02)$ & $(0.03)$ & $(-4.3)$ & $(0.5)$ \\
\hline M062X/ & 3.54 & 2.18 & 2.04 & 2.11 & 114.3 & 89.8 \\
6-31+G(d) & $(-0.12$ & $(-0.01)$ & $(-0.02)$ & $(-0.03)$ & $(-2.7)$ & $(0.3)$ \\
\hline M062X/ & 3.52 & 2.18 & 2.05 & 2.10 & 113.6 & 89.9 \\
6-31+G(d,p) & $(-0.14$ & $(-0.01)$ & $(-0.01)$ & $(-0.04)$ & $(-3.4)$ & $(0.4)$ \\
\cline { 2 - 7 }
\end{tabular}

${ }^{\mathrm{a} D e v i a t i o n}$ from the respective $\mathrm{X}$-ray value is in parentheses.

${ }^{\mathrm{b}} \mathrm{X}$ denotes $\mathrm{O}$ or $\mathrm{N}$ atom bound to $\mathrm{Mg}$.

${ }^{\mathrm{c}}$ The results from $\mathrm{B} 3 \mathrm{LYP} / 6+31+\mathrm{G}(3 \mathrm{~d}, \mathrm{p})$, which was chosen for all the calculations (numbers in bold). 
Supplementary Figure S2: Free energy profile of the $\mathrm{H}_{2} \mathrm{O} / \mathrm{OH}^{-}$exchange in 'PUNREV' with and without BSSE. The $\Delta \Delta \mathrm{G}$ free energies are plotted as a function of the effective dielectric constant $\varepsilon$. Red dots indicate $\varepsilon$ values for which a water bridge is more favorable. Figure generated using GNUplot.

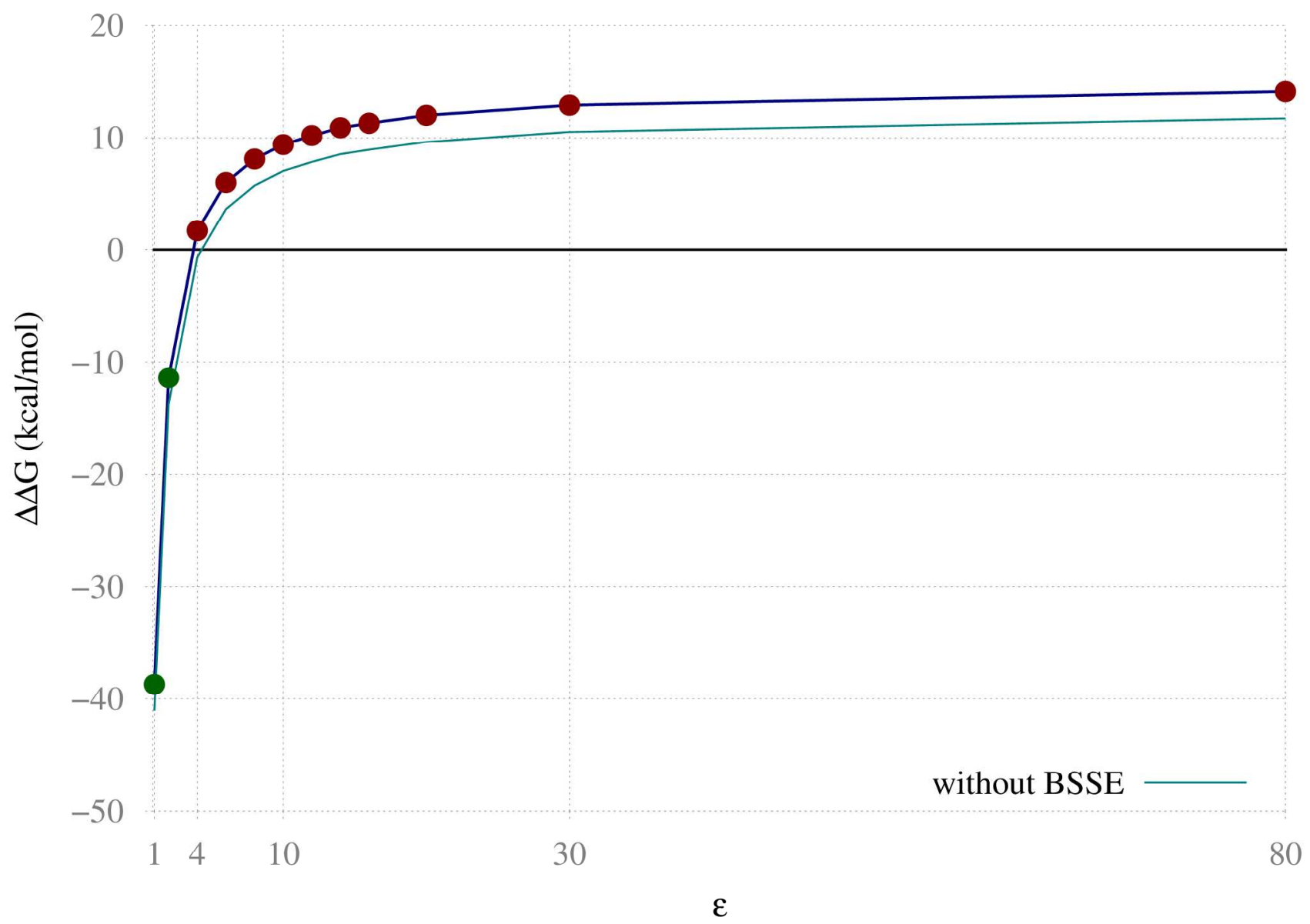


Supplementary Table S2: Experimental vs computed free energies of solvation for model ligands.

\begin{tabular}{|l|r|r|r|}
\hline & \multicolumn{3}{|c|}{$\Delta G_{\text {solv }}{ }^{80}(\mathrm{kcal} / \mathrm{mol})$} \\
\hline Metal / Ligand & Experiment & Calculated & Error \\
\hline $\mathrm{Mg}^{2+}$ & $-455.5^{\mathrm{a}}$ & -456.3 & -0.8 \\
\hline $\mathrm{H}_{2} \mathrm{O}$ & $-6.3^{\mathrm{b}}$ & -7.0 & -0.7 \\
\hline $\mathrm{OH}^{-}$ & $-105.0^{\mathrm{c}}$ & -105.6 & -0.6 \\
\hline $\mathrm{CH}_{3} \mathrm{COO}^{-}$ & $-82.2^{\mathrm{d}}$ & -82.6 & -0.4 \\
\hline $\mathrm{CH}_{3} \mathrm{COOH}$ & $-10.9^{\mathrm{e}}$ & -11.1 & -0.2 \\
\hline $\mathrm{CH}_{3} \mathrm{CONH}$ & $-10.0^{\mathrm{f}}$ & -10.5 & -0.5 \\
\hline $\mathrm{Imidazole}$ & $-10.2^{\mathrm{e}}$ & -10.6 & -0.4 \\
\hline $\mathrm{CH}_{3} \mathrm{OH}$ & $-5.1^{\mathrm{f}}$ & -6.1 & -1.0 \\
\hline $\mathrm{H}_{2} \mathrm{PO}_{4}^{-}$ & $-104.3^{\mathrm{g}}$ & -104.7 & -0.4 \\
\hline
\end{tabular}

${ }^{\mathrm{a}}$ From Friedman, 1973. ${ }^{1 \mathrm{~b}}$ From Ben-Naim, $1984 .{ }^{2 \mathrm{c}}$ From Pliego, 2000. ${ }^{3 \mathrm{~d}}$ From Lim, $1991 .{ }^{4 \mathrm{e}}$ From Wolfenden, 1981. ${ }^{5}$ From Kelly, 2006. ${ }^{6}$ From Dudev, $2006 .{ }^{7}$

1. Friedman, H. L.; Krishnan, C. V., Thermodynamics of Ionic Hydration. In Water: A Comprehensive Treatise, Franks, F., Ed. Plenum Press: New York, 1973; Vol. 3, pp 1-118. 2. Ben-Naim, A.; Marcus, Y., Solvation Thermodynamics of Nonionic Solutes. J. Chem. Phys. 1984, 81, 2016-2027.

3. Pliego, J. R., Riveros, J.M., On the Calculation of the Absolute Solvation Free Energy of Ionic Species: Application of the Extrapolation Method to the Hydroxide Ion in Aqueous Solution. J. Phys. Chem. B 2000, 104, 5155-5160.

4. Lim, C.; Bashford, D.; Karplus, M., Absolute Pka Calculations with Continuum Dielectric Methods. J. Phys. Chem. 1991, 95, 5610-5620.

5. Wolfenden, R.; Andersson, L.; Cullis, P. M.; Southgate, C. C. B., Affinities of Amino Acid Side Chains for Solvent Water. Biochemistry 1981, 20, 849-855.

6. Kelly, C. P.; Cramer, C. J.; Truhlar, D. G., Adding Explicit Solvent Molecules to Continuum Solvent Calculations for the Calculation of Aqueous Acid Dissociation Constants. J. Phys. Chem. A 2006, 110, 2493-2499.

7. Dudev, T.; Lim, C., Competition between Protein Ligands and Cytoplasmic Inorganic Anions for the Metal Cation. A Dft/Cdm Study. J. Am. Chem. Soc 2006, 128, 10541-10548. 
Supplementary Table S3: Bridge prediction for binuclear $\mathrm{Mg}^{2+}$ sites in $<2.2 \AA$ PDB structures. ${ }^{\text {a }}$

\begin{tabular}{|c|c|c|c|c|c|c|c|c|}
\hline \multirow{2}{*}{$\begin{array}{l}\text { EC } \\
\text { Number }\end{array}$} & \multirow[t]{2}{*}{ Protein Name } & \multirow[t]{2}{*}{ PDB entry } & \multirow{2}{*}{$\begin{array}{l}1^{\text {st }} \text {-shell } \\
\text { ligands }\end{array}$} & \multicolumn{2}{|c|}{$\mathrm{Q}(\mathrm{e})$} & \multicolumn{2}{|c|}{ Distance $(\AA)^{\mathrm{c}}$} & \multirow[t]{2}{*}{ Bridge } \\
\hline & & & & $1^{\text {st }}$ shell & $<8 \AA$ & $\mathrm{Mg}-\mathrm{Mg}$ & $\mathrm{Mg}-\mathrm{O}_{\mathrm{b}}$ & \\
\hline \multirow[t]{2}{*}{2.4 .2 .18} & \multirow{2}{*}{$\begin{array}{l}\text { Anthranilate } \\
\text { phosphoribosyl } \\
\text { transferase }\end{array}$} & $3 r 88$ & \multirow{2}{*}{$\begin{array}{l}\text { (E252) } \\
\text { D251* }\end{array}$} & \multirow[t]{2}{*}{+2} & \multirow[t]{2}{*}{+2} & 3.39 & 2.26 & \multirow[t]{2}{*}{$\mathrm{OH}^{-}$} \\
\hline & & $3 \mathrm{r} 6 \mathrm{c}$ & & & & 332 & 220 & \\
\hline \multirow[t]{3}{*}{2.7 .6 .3} & \multirow{3}{*}{$\begin{array}{l}\text { Pyrophospho } \\
\text { kinase }\end{array}$} & $1 \mathrm{tmj}$ & \multirow{3}{*}{$\begin{array}{l}\text { (D295) } \\
\text { (D297) } \\
\text { E16* }\end{array}$} & \multirow[t]{3}{*}{+1} & \multirow[t]{3}{*}{+2} & 3.33 & 1.92 & \multirow{3}{*}{$\mathrm{OH}^{-}$} \\
\hline & & 3hsd & & & & 3.44 & 2.29 & \\
\hline & & $1 \mathrm{~g} 4 \mathrm{c}$ & & & & 3.54 & 2.14 & \\
\hline \multirow[t]{5}{*}{2.7 .10 .1} & \multirow{5}{*}{$\begin{array}{l}\text { Tyrosine } \\
\text { kinase } \\
\text { Tyro11 }\end{array}$} & $2 \mathrm{xvd}$ & \multirow{5}{*}{$\begin{array}{l}\text { D740 } \\
\text { D759 }\end{array}$} & \multirow[t]{5}{*}{+2} & +3 & 3.27 & 1.78 & \multirow[t]{5}{*}{$\mathrm{OH}^{-}$} \\
\hline & & $2 \times 9 f$ & & & +3 & 3.25 & 1.80 & \\
\hline & & $2 \mathrm{Vx} 1$ & & & +2 & 3.28 & 1.81 & \\
\hline & & $2 \mathrm{vwz}$ & & & +2 & 3.27 & 1.78 & \\
\hline & & $2 \mathrm{vwy}$ & & & +2 & 3.40 & 1.85 & \\
\hline 2.7 .7 .52 & & $2 q 0 d$ & $\begin{array}{l}\text { ATP } \\
\text { D66* } \\
\text { D68* }\end{array}$ & -2 & -2 & 3.86 & 2.45 & $\mathrm{H}_{2} \mathrm{O}$ \\
\hline 3.4 .13 .19 & $\begin{array}{l}\text { Dipeptidase } \\
\text { AC }\end{array}$ & $3 \mathrm{fdg}$ & $\begin{array}{l}\text { E137 } \\
\text { D13 } \\
\text { H11 } \\
\text { H210 } \\
\text { H231 }\end{array}$ & +2 & +2 & 3.67 & 2.18 & $\mathrm{OH}^{-}$ \\
\hline 3.6 .1 & $\begin{array}{l}\text { U8 Snorna- } \\
\text { decapping enzyme }\end{array}$ & $2 \mathrm{xsq}$ & $\begin{array}{l}\text { E76 } \\
\text { E80 } \\
\text { G59 }\end{array}$ & +2 & +1 & 4.06 & 2.11 & $\mathrm{OH}^{-}$ \\
\hline 3.1 .3 & $\begin{array}{l}\text { Ser/Thr } \\
\text { phosphatase } \\
\text { MSPP }\end{array}$ & $2 \mathrm{v} 06$ & $\begin{array}{l}\text { D35) } \\
\text { D185 } \\
\text { D223* } \\
\text { G36 }\end{array}$ & +1 & +1 & 3.78 & 2.18 & $\mathrm{OH}^{-}$ \\
\hline 3.6 .1 & NDX2 & $2 y v m$ & $\begin{array}{l}\text { (E94) } \\
\text { E90 } \\
\text { E139 } \\
\text { A74 }\end{array}$ & +1 & 0 & 3.10 & 1.99 & $\mathrm{OH}^{-}$ \\
\hline \multirow[t]{2}{*}{3.1 .26 .4} & \multirow[t]{2}{*}{ Ribonuclease $\mathrm{H}$} & $2 \mathrm{~g} 8 \mathrm{f}$ & $\begin{array}{l}\text { (D71) } \\
\text { D132 } \\
\text { E109 } \\
\text { D192 }\end{array}$ & 0 & 0 & 3.62 & 2.02 & \multirow[t]{2}{*}{$\mathrm{OH}^{-}$} \\
\hline & & $2 \mathrm{~g} 8 \mathrm{~h}$ & D192N & +1 & 0 & 3.43 & 1.95 & \\
\hline 3.4 .13 .19 & $\begin{array}{l}\text { Xaa-Pro } \\
\text { aminopeptidase }\end{array}$ & $1 \mathrm{wl6}$ & $\begin{array}{l}\text { D271) } \\
\text { (E406) } \\
\text { E383* } \\
\text { D260 } \\
\text { H354 }\end{array}$ & 0 & 0 & 3.25 & 2.07 & $\mathrm{OH}^{-}$ \\
\hline \multirow[t]{3}{*}{3.2 .1 .143} & \multirow{3}{*}{$\begin{array}{l}\text { ADP-ribosyl } \\
\text { hydrolases }\end{array}$} & 2qty & \multirow{3}{*}{$\begin{array}{l}\text { (D300) } \\
\text { D61* } \\
\text { D18 } \\
\text { E25 } \\
\text { D62 }\end{array}$} & -1 & -2 & 3.71 & 2.19 & \multirow[t]{3}{*}{$\mathrm{H}_{2} \mathrm{O}$} \\
\hline & & $2 \mathrm{~g} 4 \mathrm{k}$ & & -1 & -2 & 3.73 & 2.16 & \\
\hline & & $2 \mathrm{foz}$ & & -1 & -2 & 3.75 & 2.17 & \\
\hline
\end{tabular}




\begin{tabular}{|c|c|c|c|c|c|c|c|c|}
\hline & & & $\begin{array}{l}\mathrm{D} 298 \\
\text { T60 } \\
\text { T301 }\end{array}$ & & & & & \\
\hline 3.1 .3 .43 & $\begin{array}{l}\text { Pyruvate } \\
\text { dehydrogenase }\end{array}$ & $2 p n q$ & $\begin{array}{l}\text { (D73) } \\
\text { D347* } \\
\text { D445 } \\
\text { G74 }\end{array}$ & +1 & -3 & 3.77 & 2.10 & $\mathrm{H}_{2} \mathrm{O}$ \\
\hline 4.6 .1 .12 & $\begin{array}{l}\text { Cyclodiphosphate } \\
\text { synthase }\end{array}$ & liv4 & $\begin{array}{l}\text { (D408) } \\
\mathrm{PO}_{4} \\
\mathrm{~K}_{3} 32 \\
\mathrm{H} 410 \\
\mathrm{H} 442\end{array}$ & 2 & -1 & 3.16 & 1.95 & $\mathrm{OH}^{-}$ \\
\hline \multirow[t]{2}{*}{ 4.2.1.11 } & $\gamma$-Enolase & 2akm & \multirow[b]{2}{*}{$\begin{array}{l}\mathrm{PO}_{4} \\
\text { D317 } \\
\text { E292 } \\
\text { D244 } \\
\text { S39 } \\
\text { S39 } \\
\end{array}$} & \multirow[t]{2}{*}{0} & \multirow[t]{2}{*}{-3} & 3.95 & 2.29 & \multirow[t]{2}{*}{$\mathrm{H}_{2} \mathrm{O}$} \\
\hline & & 1te6 & & & & 3.89 & 2.12 & \\
\hline 5.4 .99 & $\begin{array}{l}\text { Phosphate } \\
\text { synthetase }\end{array}$ & $1 \mathrm{k} 4 \mathrm{i}$ & $\begin{array}{l}\text { (E37) } \\
\mathrm{SO}_{4}{ }^{*}\end{array}$ & 2 & 0 & 3.66 & 1.95 & $\mathrm{OH}^{-}$ \\
\hline 6.1 .1 .1 & $\begin{array}{l}\text { Aspartyl tRNA- } \\
\text { synthetase }\end{array}$ & 3 nem & $\begin{array}{l}\text { (ATP) } \\
\text { E361 }\end{array}$ & -1 & -3 & 3.09 & 2.36 & $\mathrm{H}_{2} \mathrm{O}$ \\
\hline \multirow[t]{3}{*}{6.3 .3 .3} & \multirow{3}{*}{$\begin{array}{l}\text { Dethiobiotin } \\
\text { synthetase }\end{array}$} & $3 q y 0$ & \multirow{3}{*}{$\begin{array}{l}(\text { GDP*) } \\
\text { (PO4) } \\
\text { D50* } \\
\text { E116 }\end{array}$} & \multirow[t]{3}{*}{-2} & -1 & 3.57 & 2.31 & \multirow[t]{3}{*}{$\mathrm{H}_{2} \mathrm{O}$} \\
\hline & & $3 q x x$ & & & -1 & 3.58 & 2.21 & \\
\hline & & 1dak & & & -2 & 3.62 & 2.16 & \\
\hline
\end{tabular}

\footnotetext{
${ }^{a}$ The predictions are based on the guidelines summarized in Table $1 .{ }^{b}$ Residues bridging the metal ions are in parentheses, while residues hydrogen-bonding with the bridging water/hydroxide are marked by an asterisk, *; the net charge is -3 for ADP/GDP and -4 for ATP/GTP. ${ }^{c}$ Distances in italics exceed the expected distance corresponding to the bridging aqua ligand (see Table 1) by $0.05 \AA$.
} 